\title{
Dictionary Replacement for Single Image Restoration of 3D Scenes
}

T M Nimisha

ee13d037@ee.iitm.ac.in

M Arun

ee14s002@ee.iitm.ac.in

A N Rajagopalan

raju@ee.iitm.ac.in

\author{
Image Processing and Computer Vision \\ Lab \\ Indian Institute of Technology, Madras \\ Chennai, India
}

In this paper, we address the problem of jointly estimating the latent image and the depth/blur map from a single space-variantly blurred image using dictionary replacement. While most of the dictionary-based deblurring methods consider planar scenes with spaceinvariant blur, we handle 3D scenes with spacevariant blur caused by either camera motion or optical defocus. For a given blurred image, the dictionary blurred with the corresponding blur kernel provides the best representation with the least error. We formulate our problem of blur map and latent image estimation as a multi-label MRF and solve it using graph-cut.

An image $X$ degraded by space-invariant blur $h$ can be modeled by convolution as

$$
Y=h \otimes X=h \otimes D \circ \Lambda=D_{b} \circ \Lambda
$$

$h \otimes D$ is denoted as $D_{b}$, a blurred version of dictionary $D$. This implies that when kernel $h$ is known (as in non-blind deblurring), the signal $X$ can be recovered from $Y$ using the blur-invariant representation $\Lambda$. Dictionary replacement-based deblurring techniques, in fact, work on this principle.

Let $Y$ be the observed blurred image of a 3D scene and $h_{0}$ be the blur kernel corresponding to the most blurred region in the image. From the blur-depth relation, we know that the blur at any other position is a scaled down version of $h_{0}$. Hence, the problem of depth estimation boils down to estimating the scale of the blur kernel at each location. With the underlying idea that for a given sparsity, the dictionary blurred with the correct scale will represent the blurred patch with minimum error, we formulate this as a Markov Random Field (MRF) problem.

$$
\arg \min _{i} D C_{i}(k)+\sum_{k^{\prime} \in \mathcal{N}} S C\left(\bar{i}_{k^{\prime}}, i_{k}\right)
$$

where $D C_{i}(k)$ is the data cost and $S C$ is the edge aware smoothness cost.

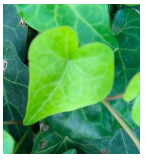

Input
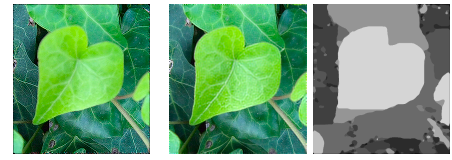

Result by [2] Our result Depthmap
We compared our method with NCSR [1], $\mathrm{Hu}$ et al. [2] (blind) and natural prior-based [3] deblurring techniques and showed that our method outperforms others both quantitatively and qualitatively. In our experiments, we included space-variant blur caused by defocus effect as well as motion blur. We also gave two applications of our method in blur magnification and image reblurring. We also considered
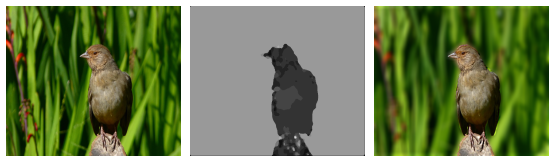

Blur Magnification
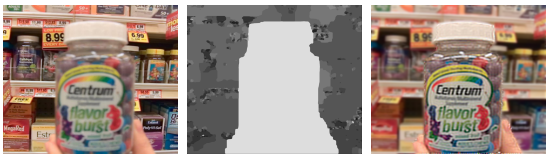

Reblurring

the case of blur due to object motion. As our method works on local patches and does not assume any global camera motion constraint, it performs well in these scenario too.

[1] Weisheng Dong, Lei Zhang, and Guangming Shi. Centralized sparse representation for image restoration. In ICCV, pages 1259-1266. IEEE, 2011.

[2] Zhe Hu, Jia-Bin Huang, and Ming-Hsuan Yang. Single image deblurring with adaptive dictionary learning. In ICIP, pages 1169-1172. IEEE, 2010.

[3] Li Xu, Shicheng Zheng, and Jiaya Jia. Unnatural 10 sparse representation for natural image deblurring. In CVPR, pages 1107-1114, 2013. 